\title{
Dihedrons of a Hyperbolic Three-Space of Positive Curvature
}

\author{
Lyudmila N. Romakina \\ (Communicated by Kazım İlarslan)
}

\begin{abstract}
We consider a hyperbolic space $\widehat{H}^{3}$ of positive curvature in the projective Cayley-Klein model. In this model the space $\widehat{H}^{3}$ is realized on the ideal domain of a Lobachevskii space $\Lambda^{3}$. This domain is an exterior of a projective space $P_{3}$ with respect to an oval surface $\gamma$ called an absolute of the spaces $\widehat{H}^{3}$ and $\Lambda^{3}$. The group $G^{3}$ of projective automorphisms of the oval surface $\gamma$ is the fundamental group of transformations for the space $\widehat{H}^{3}$ and the Lobachevskii space. In article the classification of dihedrons of the space $\widehat{H}^{3}$ is proposed. It is shown that all dihedrons of the space $\widehat{H}^{3}$ belong to fifteen types wich are invariant under the transformations of the group $G^{3}$. Dihedrons of six types are measurable by means of the absolute. Dihedrons of three types have real measures.
\end{abstract}

Keywords: hyperbolic three-space of positive curvature; dihedron of the hyperbolic three-space of positive curvature; measure of a dihedron; base of a dihedron.

AMS Subject Classification (2010): Primary: 51F10; Secondary: 51N25; 51N30.

\section{Introduction}

This paper is devoted to the 190th anniversary of non-Euclidean geometry. The first report on non-Euclidean geometry has been given at the Kazan university by Nikolay Ivanovich Lobachevsky in February, 1826. This event is considered the birth of non-Euclidean geometry.

\subsection{The hyperbolic space $\widehat{H}^{3}$ of positive curvature}

In a projective space $P_{3}$ there are three types of non-degenerate surfaces of the second order: oval surfaces; annular surfaces formed by lines; zero surfaces wich not contain the real points (see, for instance, [2, Chapter V, §15], [4, Chapter II, §4]). A signature of a quadratic form of the oval, annular, or zero surface equals two, zero, or, respectively, four. Each oval (or annular) surface divides the space $P_{3}$ into two non-homeomorphic (or, respectively, homeomorphic) domains.

In the projective Cayley-Klein model, a hyperbolic space $\widehat{H}^{3}$ of positive curvature (a complete Lobachevskii space $\Lambda^{3}$ ) is realized on the domain of the space $P_{3}$ that is exterior (interior) with respect to an oval surface $\gamma$ [14, Chapter 4, §1]. The spaces $\widehat{H}^{3}$ and $\Lambda^{3}$ are components of the expanded hyperbolic space $H^{3}$. The group $G^{3}$ of projective automorphisms of the oval surface $\gamma$ is the fundamental group of transformations for $\widehat{H}^{3}, H^{3}$, and the Lobachevskii space. The oval surface $\gamma$ is called the absolute surface or the absolute of the spaces $\widehat{H}^{3}$, $H^{3}$, and $\Lambda^{3}$.

Every line on the space $\widehat{H}^{3}$ belongs to one of three types depending on its position with respect to the absolute. Lines intersecting the absolute in two real points are called hyperbolic. If the intersection is two imaginary conjugate points, then the line is elliptic. Every tangent line to the absolute is called parabolic (Fig. 1). All flat angles in $\widehat{H}^{3}$ belong to twenty types.

Every real plane of the space $\widehat{H}^{3}$ also belongs to one of three types. An elliptic plane crosses the absolute at a zero curve (see [2, Chapter V, §15], [4, Chapter II, §4]). A hyperbolic plane of positive curvature [10], [11], [14, Chapter 4, §1] crosses the absolute at an oval curve. Every hyperbolic plane of positive curvature is one of two 
component of the expanded hyperbolic plane. A co-Euclidean plane (see [9], [13], [15]) is a tangential plane to the absolute and has a pair of imaginary conjugate lines from the absolute (see Fig. 1).

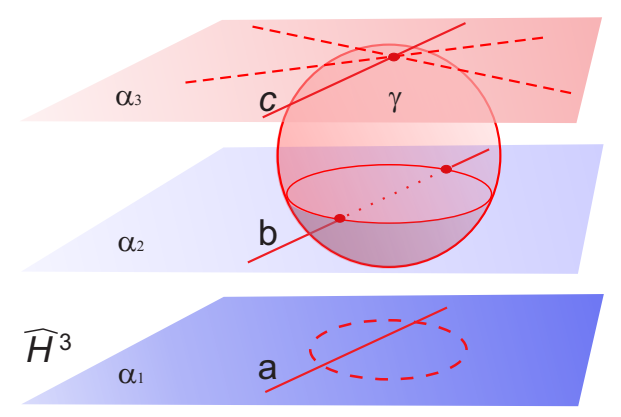

FIGURE 1. The lines and the planes on the space $\widehat{H}^{3}$ : the line $a$ is elliptic, the line $b$ is hyperbolic, the line $c$ is parabolic; the plane $\alpha_{1}$ is elliptic, the plane $\alpha_{2}$ is hyperbolic plane of positive curvature, the plane $\alpha_{3}$ is co-Euclidean

In the space $\widehat{H}^{3}$ there are also three types of planes pencils. If the axis of a planes pencil is an elliptic (hyperbolic) line, then this pencil contains two real (imaginary conjugate) tangential planes to the absolute. Such pencil is called hyperbolic (elliptic). A planes pencil with a parabolic axis contains one real tangential plane to the absolute. Such pencil is called parabolic.

\subsection{The questions of research}

In [8] the first stage of a classification of tetrahedrons in the space $\widehat{H}^{3}$ is presented. For the full classification of tetrahedrons in this space the full classification of dihedrons is necessary. We propose such classification in this article (in section 3). We show that every dihedron of the space $\widehat{H}^{3}$ belongs to one of fifteen invariant types.

By means of the absolute of the space $\widehat{H}^{3}$ we can introduce two invariant types of measurements in pencils of planes. In section 4 we introduce the dihedrons measurement in the space $\widehat{H}^{3}$ in perfect analogy to the angles measurement on the hyperbolic plane $\widehat{H}$ of positive curvature (see [7], [10]). We show that in the space $\widehat{H}^{3}$ the dihedrons of six types are measurable.

Inasmuch as in hyperbolic geometry the measurement of angles is an intricate problem, we provide all reasonings in detail. We use the principle of objects measurement in spaces with projective metrics. This principle has been created in classical works [1], [3], [4], [5]. But some stages of reasonings remain misunderstood modern researchers. As a result in non-Euclidean geometry contradictory assertions collect. In this article we suggest to return to foundations of the measurement question, to return to understand.

\section{Classification of the dihedrons of the space $\widehat{H}^{3}$}

\subsection{The pairs of planes in the space $H^{3}$}

Using for the faces types of dihedrons the designations from the work [8], we denote the type of expanded hyperbolic planes by $H$, and the type of elliptic (or co-Euclidean) planes by $E$ (or, respectively, $C$ ). The hyperbolic, elliptic, or parabolic type of a planes pencil we denote by $h, e$, or, respectively, $p$.

There are six types of orderless pairs of the planes, each of which belongs to one of three topological types $H$, $E$, and $C: H H, H E, H C, E E, E C, C C$. Disregarding realization opportunities of planes pairs in the space $H^{3}$, we obtain eighteen various sets characterizing planes pairs in pencils of three types: $H H h, H E h, H C h, E E h$, $E C h, C C h, H H e, H E e, H C e, E E e, E C e, C C e, H H p, H E p, H C p, E E p, E C p, C C p$.

Every elliptic planes pencil in $H^{3}$ contains only the expanded hyperbolic planes. Therefore the sets $H E e$, $H C e, E E e, E C e, C C e$ have not realization in the space $H^{3}$. If a pair of planes contains an elliptic plane, then this pair belongs to a hyperbolic pencil. Moreover, any two co-Euclidean planes of the space $H^{3}$ determine some hyperbolic pencil. Therefore the sets $H E p, E E p, E C p, C C p$ have not realization in $H^{3}$. Thus there are only nine sets for planes pairs in the spaces $H^{3}$ and $\widehat{H}^{3}$ :

$$
H H h, H E h, H C h, E E h, E C h, C C h, H H e, H H p, H C p .
$$




\subsection{The dihedrons types of the space $\widehat{H}^{3}$}

Any two planes of the space $\widehat{H}^{3}$ divide this space into two connected parts. We call them the dihedrons between the given planes. The planes are called faces of the dihedrons between them. Two dihedrons with the common faces are called adjacent. Let us determine the dihedrons types of the space $\widehat{H}^{3}$ in accordance with the sets from (2.1).

1. The dihedrons with the set $H H h$.

If the dihedron between two hyperbolic planes of a hyperbolic pencil does not contain (contains) the co-Euclidean planes of this pencil, we call it the hyperbolic dihedron (hyperbolic pseudodihedron).

2. The dihedrons with the set $H E h$.

A hyperbolic plane and an elliptic plane divide the space $\widehat{H}^{3}$ into two connected topologicaly equivalent parts. We call them the quasidihedrons between these planes. The quasidihedron containing (not containing) pole of the hyperbolic face with respect to the absolute is called hyperbolic (elliptic). The quasidihedron with orthogonal faces is called right.

3. The dihedrons with the set $H C h$.

Assume that $\kappa_{1}$ and $\kappa_{2}$ are co-Euclidean planes in a hyperbolic pencil and $\eta$ is a hyperbolic plane of this pencil. If the dihedron between the planes $\kappa_{1}$ and $\eta$ does not contain (contains) the plane $\kappa_{2}$, we call it the hyperbolic dihedral flag (hyperbolic dihedral pseudoflag) with the fases $\kappa_{1}$ and $\eta$.

4. The dihedrons with the set $E E h$.

If the dihedron between two elliptic planes does not contain (contains) the absolute, we call it the elliptic dihedron (elliptic pseudodihedron).

5. The dihedrons with the set $E C h$.

If the dihedron between an elliptic plane and a co-Euclidean plane does not contain (contains) the absolute hyperquadric, we call it the elliptic dihedral flag (elliptic dihedral pseudoflag).

6. The dihedrons with the set $C C h$.

If the dihedron between two co-Euclidean planes does not contain (contains) the absolute hyperquadric, we call it the dihedral valiana (dihedral covaliana).

7. The dihedron with the set $H H e$.

Two hyperbolic planes of an elliptic pencil divide the space $\widehat{H}^{3}$ into two topologicaly equivalent parts. We call them the semispaces between the given planes.

8. The dihedrons with the set $H H p$.

If the dihedron between the hyperbolic planes of a parabolic pencil does not contain (contains) the coEuclidean plane of this pencil, we call it the layer (pseudolayer) between the given planes.

9. The dihedrons with the set $H C p$.

A hyperbolic plane and a co-Euclidean plane of a parabolic pencil divide the space $\widehat{H}^{3}$ into two topologicaly equivalent parts. We call them the dihedral parabolic flags between the given planes.

Thus all admissible pairs of planes determine fifteen dihedrons types in the space $\widehat{H}^{3}$. We represent all dihedrons types in the table 1.

\section{The dihedrons measurement in the space $\widehat{H}^{3}$}

\subsection{Principles of measurement}

In spaces with projective metrics, a measurement of objects determined by two elements of some pencil is called hyperbolic (elliptic) if it is set by means of the pair of absolute real (imaginary conjugate) elements of the given pencil. A parabolic measurement is set by means of the pair of real coincided elements (see, for instance, 
Table 1. The types and measures of dihedrons of the space $\widehat{H}^{3}$

\begin{tabular}{|c|c|c|c|c|}
\hline \multirow[t]{2}{*}{ Type of a dihedron } & \multirow[t]{2}{*}{$\begin{array}{l}\text { Measure } v(\tilde{v}) \text { of } \\
\text { a dihedron }\end{array}$} & \multirow{2}{*}{$\begin{array}{l}\text { Type of } \\
\text { a faces } \\
\text { pencil }\end{array}$} & \multicolumn{2}{|c|}{$\begin{array}{l}\text { Types } \\
\text { of faces }\end{array}$} \\
\hline & & & $\alpha$ & $\beta$ \\
\hline Dihedral valiana & - & \multirow[t]{2}{*}{$h$} & \multirow[t]{2}{*}{$C$} & \multirow[t]{2}{*}{$C$} \\
\hline Dihedral covaliana & - & & & \\
\hline Dihedral hyperbolic flag & - & \multirow[t]{2}{*}{$h$} & \multirow{3}{*}{$C$} & \multirow{3}{*}{$H$} \\
\hline Dihedral hyperbolic pseudoflag & - & & & \\
\hline Dihedral parabolic flag & - & $p$ & & \\
\hline Dihedral elliptic flag & - & \multirow[t]{2}{*}{$h$} & \multirow[t]{2}{*}{$C$} & \multirow[t]{2}{*}{$E$} \\
\hline Dihedral elliptic pseudoflag & - & & & \\
\hline Semispace & $v \in[0 ; \pi]$ & $e$ & \multirow{5}{*}{$H$} & \multirow{5}{*}{$H$} \\
\hline Hyperbolic dihedron & $v \in \mathbb{R}_{+}$ & \multirow[t]{2}{*}{$h$} & & \\
\hline Hyperbolic pseudodihedron & $\tilde{v}=i \pi+v, v \in \mathbb{R}_{+}$ & & & \\
\hline Layer & - & \multirow[t]{2}{*}{$p$} & & \\
\hline Pseudolayer & - & & & \\
\hline Quasidihedron & $\begin{array}{c}\tilde{v}=\varepsilon v+i \pi / 2, \\
v \in \mathbb{R}_{+} \\
\varepsilon=1 ;-1 ; 0\end{array}$ & $h$ & $H$ & $E$ \\
\hline Elliptic dihedron & $v \in \mathbb{R}_{+}$ & $h$ & $E$ & $E$ \\
\hline Elliptic pseudodihedron & $\tilde{v}=i \pi-v, v \in \mathbb{R}_{+}$ & & & \\
\hline
\end{tabular}

[4, Chapter VI, §1], [6, Part III, §20.3]). In other words, the measurement type in a pencil is identical to the type of this pencil. We notice that a figure consisting of all points on a line is also called a pencil of points (see, for instance, [16, Chapter IX]). We adhere here to traditional names.

Let $\alpha$ and $\beta$ be planes of the space $\widehat{H}^{3}$ and $k=\alpha \cap \beta$. Let us denote the planes pencil with axis $k$ by $\Theta$. The pencil $\Theta$ contains two co-Euclidean planes. We denote them by $\kappa_{1}$ and $\kappa_{2}$. The pencil $\Theta$ is parabolic if and only if $\kappa_{1}=\kappa_{2}$.

The cross-ratio of a quadruple of planes from a pencil is an invariant of all projective transformations. Hence the cross-ratio $\left(\alpha \beta \kappa_{1} \kappa_{2}\right)$ is an invariant of the fundamental group $G^{3}$ of the space $\widehat{H}^{3}$. The type of a dihedron is an invariant of the group $G^{3}$ too. Therefore we express the measure of the dihedron between the planes $\alpha$ and $\beta$ through the cross-ratio $\left(\alpha \beta \kappa_{1} \kappa_{2}\right)$.

Let us consider all possibilities.

1. Assume that the faces $\alpha$ and $\beta$ of the dihedron are non-co-Euclidean planes and the pencil $\Theta$ containing the faces is non-parabolic.

If the pencil $\Theta$ is hyperbolic (or elliptic), then the planes $\kappa_{1}$ and $\kappa_{2}$ are real (or, respectively, imaginary conjugate). In this case by means of the cross-ratio $\left(\alpha \beta \kappa_{1} \kappa_{2}\right)$ it is possible to set the hyperbolic (or, respectively, elliptic) measurement of dihedrons with axis $k$. Hence in the space $\widehat{H}^{3}$ the following dihedrons are measurable: semispace, quasidihedron, hyperbolic dihedron, hyperbolic pseudodihedron, elliptic dihedron, and elliptic pseudodihedron.

2. Assume that the faces $\alpha$ and $\beta$ of the dihedron are non-co-Euclidean planes and the pencil $\Theta$ containing the faces is parabolic.

In this case the faces $\alpha$ and $\beta$ of the dihedron are the hyperbolic planes and $\kappa_{1}=\kappa_{2}$. Therefore we have $\left(\alpha \beta \kappa_{1} \kappa_{2}\right)=1$. It means that any two pairs of the hyperbolic planes from a parabolic pencil are congruent to each other. Consequently, layers and pseudolayers are immeasurable dihedrons in the space $\widehat{H}^{3}$.

If we consider a parabolic pencil of planes as a limiting position of a hyperbolic pencil of planes, then we can define artificial measures of layers and pseudolayers (see analogous reasonings for angles of the plane $\widehat{H}$ in [12]).

3. Assume that at least one of the faces $\alpha$ and $\beta$ of the dihedron is the co-Euclidean plane.

Let the face $\alpha$ be a co-Euclidean plane. Then $\alpha$ coincides at least with one of the planes $\kappa_{1}, \kappa_{2}$. In this case the number $\left(\alpha \beta \kappa_{1} \kappa_{2}\right)$ is not defined. Consequently, in the space $\widehat{H}^{3}$ the following dihedrons 
are immeasurable: dihedral valiana, dihedral covaliana, dihedral hyperbolic flag, dihedral hyperbolic pseudoflag, dihedral elliptic flag, and dihedral elliptic pseudoflag.

We notice that any two measurable dihedrons are congruous if and only if they belong to one type and have equal measures. Any two immeasurable dihedrons of one type are congruous.

In subsections 3.2-3.5 we define the measures of the measurable dihedrons of the space $\widehat{H}^{3}$. We need the following properties of points of an elliptic (hyperbolic) line.

Lemma 3.1. Two orthogonal points divide the elliptic line containing them into two congruous segments.

Lemma 3.2. Let $A$ and $B$ be distinct non-orthogonal points of an elliptic line l. If a point $A^{\prime}\left(B^{\prime}\right)$ is orthogonal on the line l to the point $A(B)$, then the points $A^{\prime}$ and $B^{\prime}$ belong to one segment between the points $A$ and $B$.

Lemma 3.3. Two orthogonal points divide the hyperbolic line containing them into two congruous quasisegments.

Lemma 3.4. Let $A$ and $B$ be non-orthogonal points on various branches of a hyperbolic line l. If a point $A^{\prime}\left(B^{\prime}\right)$ is orthogonal on the line $l$ to the point $A(B)$, then the points $A^{\prime}$ and $B^{\prime}$ belong to different quasisegments between the points $A$ and $B$.

Proofs of these properties are offered, for example, in [10, Lemmas 4.2.1-4.2.4], where elliptic and hyperbolic lines are considered in the hyperbolic plane $\widehat{H}$ of positive curvature. But the given properties do not depend on a type of the space containing the considered lines. Therefore we have provided here more general formulations of these properties.

\subsection{The measure of a semispace}

Assume that hyperbolic planes $\alpha$ and $\beta$ of the elliptic pencil $\Theta$ form adjacent semispaces $\nu_{1}$ and $\nu_{2}$. The pencil $\Theta$ with the hyperbolic axis $k=\alpha \cap \beta$ contains two imaginary conjugate tangential planes to the absolute. We denote them by $\kappa_{1}$ and $\kappa_{2}$. For the planes $\alpha, \beta, \kappa_{1}$, and $\kappa_{2}$ we have $\left(\alpha \beta \kappa_{1} \kappa_{2}\right) \in \mathbb{C}$ and $\left|\left(\alpha \beta \kappa_{1} \kappa_{2}\right)\right|=1$ (see the similar proof in [10, Theorem 1.11.2]).

Let us consider the number

$$
v=\left|\frac{1}{2 i} \ln \left(\alpha \beta \kappa_{1} \kappa_{2}\right)\right|,
$$

where the function $\ln z$ is the principal value of the complex logarithm $\operatorname{Ln} z$ of $z=\left(\alpha \beta \kappa_{1} \kappa_{2}\right)$. The function $\ln z$ is defined by the condition

$$
\ln z=\ln |z|+i \arg (z), \quad-\pi<\arg (z) \leq \pi .
$$

Using the equality from (3.2), we find

$$
v=\left|\frac{1}{2 i}\left[\ln \left|\left(\alpha \beta \kappa_{1} \kappa_{2}\right)\right|+i \arg \left(\alpha \beta \kappa_{1} \kappa_{2}\right)\right]\right|=\frac{1}{2}\left|\arg \left(\alpha \beta \kappa_{1} \kappa_{2}\right)\right| .
$$

Hence $v \in[0 ; \pi / 2]$. Thus the multiplier $1 / 2 i$ in the formula (3.1) allows us to obtain the real measure $v$ of a semispace, using the complex number $\left(\alpha \beta \kappa_{1} \kappa_{2}\right)$. The expression (3.1) is the Laguerre formula adapted to measurement of dihedrons of concrete type. This formula is universal in elliptic measurement of objects (see, for instance, [4, Chapter VI, §1], [6, Part III, §20.3]).

Suppose the planes $\alpha$ and $\alpha^{\prime}$ of the elliptic pencil $\Theta$ are orthogonal. It means that $\left(\alpha \alpha^{\prime} \kappa_{1} \kappa_{2}\right)=-1$. Then by the principle of duality of the space $\widehat{H}^{3}$ from Lemma 3.1 we conclude that the planes $\alpha$ and $\alpha^{\prime}$ divide the space $\widehat{H}^{3}$ into two congruous semispaces. We call them the right semispaces. The value $v$ from (3.1) for the orthogonal planes $\alpha$ and $\alpha^{\prime}$ equals $\pi / 2$. Thus the number $\pi / 2$ corresponds to every right semispace. We call this number the measure of a right semispace.

If the planes $\alpha$ and $\beta$ are not orthogonal, then we consider the planes $\alpha^{\prime}$ and $\beta^{\prime}$ wich satysfy the following conditions:

$$
\alpha^{\prime} \perp \alpha, \quad k \subset \alpha^{\prime}, \quad \beta^{\prime} \perp \beta, \quad k \subset \beta^{\prime} .
$$

By the principle of duality of the space $\widehat{H}^{3}$ from Lemma 3.2 we conclude that the planes $\alpha^{\prime}$ and $\beta^{\prime}$ belong to one semispace between the planes $\alpha$ and $\beta$. The semispace containing (not containing) the planes $\alpha^{\prime}$ and $\beta^{\prime}$ is called wide (narrow).

We may consider the space $\widehat{H}^{3}$ as the sum of two adjacent right semispaces. Hence the summary measure of the adjacent semispaces $\nu_{1}$ and $\nu_{2}$ between the planes $\alpha$ and $\beta$ equals $\pi$. For this reason we call the value $v$ from (3.1) (or $\pi-v$ ) the measure of the narrow (or, respectively, wide) semispace between the planes $\alpha$ and $\beta$. 
The measure of the semispace between the planes $\alpha, \beta$ does not depend on a sequence order of the planes in the pairs $\alpha, \beta$ and $\kappa_{1}, \kappa_{2}$. Indeed, for the cross-ratio $\left(\alpha \beta \kappa_{1} \kappa_{2}\right)$ we have

$$
\left(\beta \alpha \kappa_{1} \kappa_{2}\right)=\left(\alpha \beta \kappa_{1} \kappa_{2}\right)^{-1}, \quad\left(\alpha \beta \kappa_{2} \kappa_{1}\right)=\left(\alpha \beta \kappa_{1} \kappa_{2}\right)^{-1} .
$$

Consequently,

$$
\left|\frac{1}{2 i} \ln \left(\beta \alpha \kappa_{1} \kappa_{2}\right)\right|=\left|\frac{1}{2 i} \ln \left(\alpha \beta \kappa_{2} \kappa_{1}\right)\right|=\left|\frac{1}{2 i} \ln \left(\alpha \beta \kappa_{1} \kappa_{2}\right)\right|=v .
$$

In [10, Theorem 4.4.1] it is proved that the length of a segment on an elliptic line in the plane $\widehat{H}$ is additive. Owing to this fact on the principle of duality of the space $\widehat{H}^{3}$ the entered measure of a semispace is additive too.

\subsection{The measure of an elliptic (hyperbolic) dihedron}

3.3.1. Definitions. Assume that elliptic planes $\alpha$ and $\beta$ form the elliptic dihedron $\nu$ with an elliptic axis $k$. Let $\kappa_{1}$ and $\kappa_{2}$ be the co-Euclidean planes of the hyperbolic pencil $\Theta$ with the axis $k$. By the definition of an elliptic dihedron the pairs of the planes $\alpha, \beta$ and $\kappa_{1}, \kappa_{2}$ do not divide each other. Consequently, we have $\left(\alpha \beta \kappa_{1} \kappa_{2}\right) \in \mathbb{R}_{+}$. Owing to this condition the number

$$
v=\frac{1}{2}\left|\ln \left(\alpha \beta \kappa_{1} \kappa_{2}\right)\right|
$$

is real and positive too. We call it the measure of the elliptic dihedron $\nu$.

Now suppose the hyperbolic planes $\alpha$ and $\beta$ of the hyperbolic pencil form the hyperbolic dihedron $\bar{\nu}$. The planes $\alpha$ and $\beta$ in the pencil with the elliptic axis $k$ do not divide the pair of the co-Euclidean planes $\kappa_{1}$ and $\kappa_{2}$ of this pencil. Hence $\left(\alpha \beta \kappa_{1} \kappa_{2}\right) \in \mathbb{R}_{+}$.

We call the number $v$ from (3.3) the measure of the hyperbolic dihedron $\bar{\nu}$. The number $(-v)$ is the agreed measure of this dihedron.

The measure of the elliptic (hyperbolic) dihedron between the planes $\alpha$ and $\beta$ does not depend on a sequence order of the planes in the pairs $\alpha, \beta$ and $\kappa_{1}, \kappa_{2}$. Using the approach offered in the proof of Theorem 4.4.2 from [10], we can prove that the entered measure of an elliptic (hyperbolic) dihedron is additive.

3.3.2. Remark about the Laguerre formula. The way of calculation of angles measures on the Euclidean plane by means of projective geometry is established in [5]. The measurement of angles on the Euclidean plane is elliptic. Therefore the formula obtained by Laguerre is suitable only to elliptic pencils. Generalization of the Laguerre formula for hyperbolic pencils has become possible on account of works by Cayley and Klein.

The choice principle of a constant in the Laguerre formula is in detail considered by Klein in [3], [4] (see also [6], [10]). This principle at calculation of the lengths of segments on hyperbolic lines does not raise doubts of researchers. But at calculation of angular measures in hyperbolic pencils some authors trespass against this principle. It leads to the wrong results. Therefore we pay attention to an important detail of reasonings. Since for elliptic and hyperbolic dihedrons the number $\left(\alpha \beta \kappa_{1} \kappa_{2}\right)$ is real and positive, in the Laguerre formula (3.3) it is necessary to accept the multiplier $1 / 2$. This real multiplier via the formula (3.3) provides transition from the real positive number $\left(\alpha \beta \kappa_{1} \kappa_{2}\right)$ to the real positive measure $v$ of the dihedron.

Moreover, the universal choice of the constant in the Laguerre formula for pencils of one type provides the uniform logical scheme for creation of different non-Euclidean geometries.

3.3.3. Measures of conjugate dihedrons. If elliptic planes $\alpha, \beta$ and hyperbolic planes $\alpha^{\prime}, \beta^{\prime}$ in a hyperbolic planes pencil $\Theta$ satisfy the conditions $\alpha \perp \alpha^{\prime}$ and $\beta \perp \beta^{\prime}$, then the elliptic dihedron between the planes $\alpha$, $\beta$ and the hyperbolic dihedron between the planes $\alpha^{\prime}, \beta^{\prime}$ are called conjugate to each other.

Let $\kappa_{1}$ and $\kappa_{2}$ be the co-Euclidean planes of the pencil $\Theta$. Since

$$
\alpha \perp \alpha^{\prime} \Longleftrightarrow\left(\alpha \alpha^{\prime} \kappa_{1} \kappa_{2}\right)=-1, \quad \beta \perp \beta^{\prime} \Longleftrightarrow\left(\beta \beta^{\prime} \kappa_{1} \kappa_{2}\right)=-1,
$$

by properties of the cross-ratio of a quadruple of planes from a pencil we have

$$
\left(\alpha^{\prime} \beta^{\prime} \kappa_{1} \kappa_{2}\right)=\left(\alpha^{\prime} \alpha \kappa_{1} \kappa_{2}\right)\left(\alpha \beta^{\prime} \kappa_{1} \kappa_{2}\right)=-\left(\alpha \beta^{\prime} \kappa_{1} \kappa_{2}\right)=-\left(\alpha \beta \kappa_{1} \kappa_{2}\right)\left(\beta \beta^{\prime} \kappa_{1} \kappa_{2}\right)=\left(\alpha \beta \kappa_{1} \kappa_{2}\right) .
$$

Therefore the measures of conjugate dihedrons are equal. 


\subsection{The measure of a quasidihedron}

Assume that a hyperbolic plane $\alpha$ and an elliptic plane $\beta$ form adjacent quasidihedrons $\nu_{1}$ and $\nu_{2}$. The planes $\alpha$ and $\beta$ in the pencil $\Theta$ divide the pair of the co-Euclidean planes $\kappa_{1}$ and $\kappa_{2}$. Hence $\left(\alpha \beta \kappa_{1} \kappa_{2}\right) \in \mathbb{R},\left(\alpha \beta \kappa_{1} \kappa_{2}\right)<0$, and $\arg \left(\alpha \beta \kappa_{1} \kappa_{2}\right)=\pi$. We pressume that

$$
v=\frac{1}{2} \ln \left(\alpha \beta \kappa_{1} \kappa_{2}\right),
$$

where the function $\ln z$ is defined by condition (3.2).

From (3.4) via the condition (3.2) we obtain $v=\left[\ln \left|\left(\alpha \beta \kappa_{1} \kappa_{2}\right)\right|+i \pi\right] / 2$. Consequently, $v \in \mathbb{C}$ and $\operatorname{Im}(v)=\pi / 2$.

We consider the hyperbolic plane $\beta^{\prime}$ orthogonal to the given elliptic plane $\beta$ in the pencil $\Theta$. By the principle of duality of the space $\widehat{H}^{3}$ from Lemma 3.3 we conclude that the planes $\beta, \beta^{\prime}$ divide the space $\widehat{H}^{3}$ into two congruous quasidihedrons. We call them the right quasidihedrons. For the pairs of the planes $\beta$ and $\beta^{\prime}$ the number $v$ from (3.4) equals $i \pi / 2$. In view of this we call the number $i \pi / 2$ the measure of a right quasidihedron.

The space $\widehat{H}^{3}$ can be considered as the sum of two adjacent quasidihedrons, in particular, of two adjacent right quasidihedrons. Therefore we appropriate the measure $i \pi$ to the space $\widehat{H}^{3}$ accepted as a dihedron of a hyperbolic planes pencil.

The numberes $v$ and $i \pi-v$, that is, the numberes

$$
\pm \frac{1}{2}|\ln |\left(\alpha \beta \kappa_{1} \kappa_{2}\right)||+i \frac{\pi}{2},
$$

are called the measures of the adjacent quasidihedrons $\nu_{1}$ and $\nu_{2}$ between the planes $\alpha$ and $\beta$.

The entered measure of the quasidihedron between the planes $\alpha$ and $\beta$ does not depend on a sequence order of the planes in pairs $\alpha, \beta$ and $\kappa_{1}, \kappa_{2}$. The sum of quasidihedrons is not a quasidihedron. For this reason the question of additivity of a measure for a quasidihedron does not make sense.

Suppose the planes $\alpha^{\prime}, \beta^{\prime}$ in the pencil $\Theta$ satisfy the following conditions: $\alpha^{\prime} \perp \alpha, \beta^{\prime} \perp \beta$. According to Lemma 3.4 by the principle of duality of the space $\widehat{H}^{3}$ the planes $\alpha^{\prime}$ and $\beta^{\prime}$ belong to various quasidihedrons between the planes $\alpha$ and $\beta$. Let $\nu_{1}\left(\nu_{2}\right)$ be the hyperbolic (elliptic) quasidihedron between the planes $\alpha$ and $\beta$. Then the quasidihedron $\nu_{1}\left(\nu_{2}\right)$ contains the plane $\alpha^{\prime}\left(\beta^{\prime}\right)$. Therefore the quasidihedron $\nu_{1}\left(\nu_{2}\right)$ consists from the right quasidihedron $\sigma_{\alpha}\left(\sigma_{\beta}\right)$ between the planes $\alpha, \alpha^{\prime}\left(\beta, \beta^{\prime}\right)$ of the measure $i \pi / 2$ and the elliptic (hyperbolic) dihedron $\sigma_{\mathrm{e}}\left(\sigma_{\mathrm{h}}\right)$ between the planes $\alpha^{\prime}, \beta\left(\beta^{\prime}, \alpha\right)$. We denote the measure (agreed measure) of the elliptic (hyperbolic) dihedron $\sigma_{\mathrm{e}}\left(\sigma_{\mathrm{h}}\right)$ by a simbol $\alpha_{\mathrm{e}}\left(\alpha_{\mathrm{h}}\right)$. By the definition of the measure (agreed measure) of an elliptic (hyperbolic) dihedron we have $\alpha_{\mathrm{e}} \in \mathbb{R}$ and $\alpha_{\mathrm{e}}>0\left(\alpha_{\mathrm{h}} \in \mathbb{R}\right.$ and $\left.\alpha_{\mathrm{h}}<0\right)$.

We display the section of the discussion objects by some hyperbolic plane of the space $\widehat{H}^{3}$ in Fig. 2 .

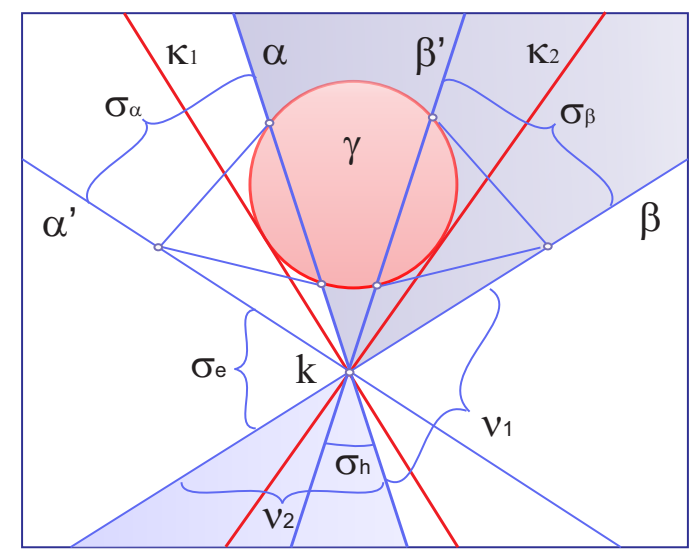

FIGURE 2. The section of the quasidihedrons $\nu_{1}$ and $\nu_{2}$ of the space $\widehat{H}^{3}$ by some hyperbolic plane

The sum of the measures $v_{1}$ and $v_{2}$ of the adjacent quasidihedrons $\nu_{1}$ and $\nu_{2}$ equals $i \pi$. Hence

$$
v_{1}+v_{2}=i \frac{\pi}{2}+\alpha_{\mathrm{e}}+i \frac{\pi}{2}+\alpha_{\mathrm{h}}=i \pi \text {. }
$$

It follows that $\alpha_{\mathrm{h}}=-\alpha_{\mathrm{e}}$ and

$$
v_{1}=i \frac{\pi}{2}+\alpha_{\mathrm{e}}, \quad v_{2}=i \frac{\pi}{2}-\alpha_{\mathrm{e}}
$$


Taking into account these equalities we formulate the following definitions.

We call the number

$$
\frac{i \pi}{2}+\frac{1}{2}|\ln |\left(\alpha \beta \kappa_{1} \kappa_{2}\right)|| \quad\left(\frac{i \pi}{2}-\frac{1}{2}|\ln |\left(\alpha \beta \kappa_{1} \kappa_{2}\right)||\right)
$$

the measure of the hyperbolic (or, respectively, elliptic) quasidihedron between the planes $\alpha$ and $\beta$.

\subsection{The measure of an elliptic (hyperbolic) pseudodihedron}

Assume that elliptic planes $\alpha$ and $\beta$ form two adjacent dihedrons: the elliptic dihedron $\nu_{\mathrm{e}}$ of the measure $v$ from (3.3), where $v \in \mathbb{R}_{+}$, and the elliptic pseudodihedron $\psi_{\mathrm{e}}$ (Fig. 3). The elliptic pseudodihedron $\psi_{\mathrm{e}}$ consists from two right quasidihedrons and the hyperbolic dihedron $\nu_{\mathrm{h}}$ conjugate to $\nu_{\mathrm{e}}$. The agreed measure of the hyperbolic dihedron $\nu_{\mathrm{h}}$ equals $(-v)$. Owing to this we call the number $i \pi-v$ the measure of the elliptic pseudodihedron $\psi_{\mathrm{e}}$.

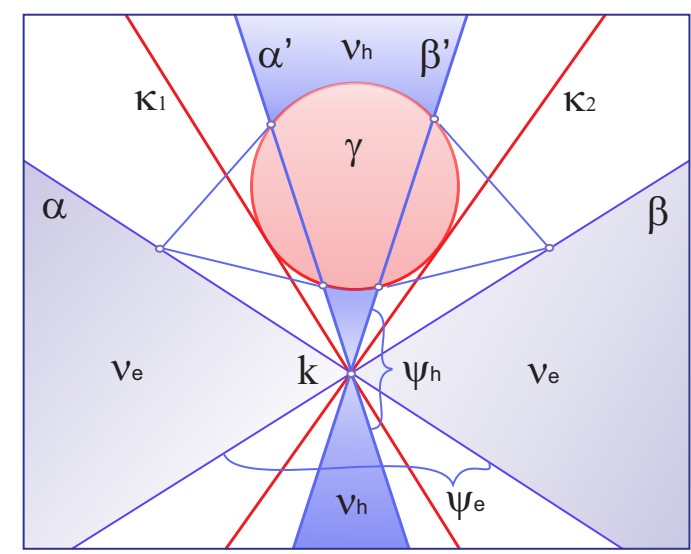

FIGURE 3. The section of the dihedrons $\nu_{\mathrm{e}}$ and $\nu_{\mathrm{h}}$ in the space $\widehat{H}^{3}$ by some hyperbolic plane

Let now $\alpha^{\prime}$ and $\beta^{\prime}$ be the given hyperbolic planes. These planes form two adjacent dihedrons: the hyperbolic dihedron $\nu_{\mathrm{h}}$ of measure $v$ (3.3), where $v \in \mathbb{R}_{+}$, and the hyperbolic pseudodihedron $\psi_{\mathrm{h}}$ (see Fig. 3). The hyperbolic pseudodihedron $\psi_{\mathrm{h}}$ consists from two right quasidihedrons and the elliptic dihedron $\nu_{\mathrm{e}}$ of the measure $v$. The elliptic dihedron $\nu_{\mathrm{e}}$ is conjugate to the hyperbolic dihedron $\nu_{\mathrm{h}}$. Owing to this we call the number $i \pi+v$ the measure of the hyperbolic pseudodihedron $\psi_{\mathrm{h}}$. As the agreed measure of the hyperbolic dihedron $\nu_{\mathrm{h}}$ equals $v_{0}$, where $v_{0}=-v$, the measure of the hyperbolic pseudodihedron $\psi_{\mathrm{h}}$ equals $i \pi-v_{0}$.

Finishing a discourse about the measurement of dihedrons in $\widehat{H}^{3}$, we notice that the formally calculated measures of layers and pseudolayers equal zero (see the formula (3.3) on the condition $\kappa_{1}=\kappa_{2}$ ). In some tasks we accept the numberes 0 and $i \pi$ as the artificial measures of layers and pseudolayers, respectively (see [12]).

Outcomes of reasonings are presented in the table 1.

\subsection{The linear measure of a dihedron of the space $\widehat{H}^{3}$}

Let $\alpha$ and $\beta$ be faces of some dihedron $F$ of the space $\widehat{H}^{3}$. The pole of the plane $\alpha(\beta)$ with respect to the absolute we denote by $S_{\alpha}\left(S_{\beta}\right)$. Let $A=\alpha \cap S_{\alpha} S_{\beta}$ and $B=\beta \cap S_{\alpha} S_{\beta}$. The line $S_{\alpha} S_{\beta}$ is a common perpendicular of the planes $\alpha$ and $\beta$. If $k=\alpha \cap \beta$, then the line $S_{\alpha} S_{\beta}$ is the polar of the line $k$ with respect to the absolute. One part of the line $S_{\alpha} S_{\beta}$ between the points $A$ and $B$ belongs to the dihedron $F$. We call this part a base of the dihedron $F$.

Suppose $F$ is the measurable dihedron. Then $S_{\alpha} S_{\beta}$ and $k$ are non-parabolic lines of the various types and $A B$ is a segment or a quasisegment of the line $S_{\alpha} S_{\beta}$.

The pencil $\Theta$ with the axis $k$ contains two tangent planes to the absolute. We denote them by $\kappa_{1}$ and $\kappa_{2}$. Let $K_{1}=\kappa_{1} \cap \gamma$ and $K_{2}=\kappa_{2} \cap \gamma$. The measurement in the pencil $\Theta$ is set by means of the planes $\kappa_{1}$ and $\kappa_{2}$. The measurement on the line $S_{\alpha} S_{\beta}$ is set by means of the points $K_{1}$ and $K_{2}$. By definition in the Laguerre formula the constant multiplier of the measure $v$ of the dihedron $F$ is equal to $1 / 2 \tau$, where $\tau=i(\tau=1)$ for the elliptic (hyperbolic) pencil $\Theta$. The length $|A B|$ of the segment or the quasisegment $A B$ by definition can 
be calculated via the similar formula with the constant multiplier $\rho / 2 \tau$, where $\tau=i(\tau=1)$ for the elliptic (hyperbolic) line $S_{\alpha} S_{\beta}[10, \S 4.4]$. The pencils $\Theta$ and $S_{\alpha} S_{\beta}$ of one type. Moreover, accoding to the construction we have $\left(\alpha \beta \kappa_{1} \kappa_{2}\right)=\left(A B K_{1} K_{2}\right)$. Consequently, $v=|A B| / \rho$.

Thus we proved the following assertion: The measure of a measurable dihedron in the space $\widehat{H}^{3}$ is equal to the relation of the length of its base to the curvature radius $\rho$ of the space $\widehat{H}^{3}$.

The similar result for the plane $\widehat{H}$ is proved in [10, Theorem 4.7.1]: The measure of a measurable angle of the plane $\widehat{H}$ is equal to the relation of the length of its base to the curvature radius $\rho$ of the plane $\widehat{H}$.

\section{Conclusion}

In this work we have geometrically defined types of dihedrons in the hyperbolic space $\widehat{H}^{3}$ of positive curvature and have offered geometrical ways of their measurement. We proved that by means of the absolute of the space $\widehat{H}^{3}$ it is possible to measure dihedrons of six of fifteen types. In the accepted definitions the dihedrons of three types have real measures. At the following stage of researches we will express the entered measures of the dihedrons through coordinates of faces. Inasmuch as in the space $\widehat{H}^{3}$ it is possible to use various convenient coordinate systems, we will derive formulae of expression of measures of the dihedrons in two most convenient canonical frames.

\section{References}

[1] Cayley A., A Sixth memoir upon quantics. Phil. Trans. Roy. Soc. London. 149 (1859).

[2] Efimov N. V., Higher Geometry. MAIK. Nauka/Interperiodika. FIZMATLIT, Moscow, 2004. (In Russian)

[3] Klein F., Vergleichende Betrachtungen über neuere geometrische Forschungen, Programm zum Eintritt in die philosophische Facultat und den Senat der Universitat zu Erlangen. A. Deichert, Erlangen, 1872.

[4] Klein F., Vorlesungen Über Nicht-Euclidische Geometrie. Verlag von Julius Springer, Berlin, 1928.

[5] Laguerre, Sur la theorie des foyers. Nouv. Ann. de Mathem. 12 (1853), 57-66.

[6] Richter-Gebert Jürgen, Perspectives on Projective Geometry: A Guided Tour Through Real and Complex. Springer Science + Business Media, New York, 2011.

[7] Romakina L. N., Analogs of a formula of Lobachevsky for angle of parallelism on the hyperbolic plane of positive curvature. Sib. Elektron. Mat. Izv. 10 (2013), 393-407. (In Russian)

[8] Romakina L. N., Classification of tetrahedrons with not hyperbolic sides in a hyperbolic space of positive curvature. Chebyshevskii Sb. 16 (2015), no. 2, 208-221. (In Russian)

[9] Romakina L. N., Geometries of the co-Euclidean and co-pseudoeuclidean planes. Saratov, Publishing house Scientific book, 2008. (In Russian)

[10] Romakina L. N., Geometry of the hyperbolic plane of positive curvature. P. 1: Trigonometry. Saratov, Publishing House of the Saratov University, 2013. (In Russian)

[11] Romakina L. N., Geometry of the hyperbolic plane of positive curvature. P. 2: Transformations and simple partitions. Saratov, Publishing House of the Saratov University, 2013. (In Russian)

[12] Romakina L. N., The Area of a Generalized Polygon without Parabolic Edges of a Hyperbolic Plane of Positive Curvature. Asian Journal of Mathematics and Computer Research 10 (2016), no. 4, 293-310.

[13] Rosenfeld B. A., Geometry Of Lie Groups. Springer Science + Business Media, New York, 2015.

[14] Rosenfeld B. A., Non-Euclidean spaces. Nauka, Moscow, 1969. (In Russian)

[15] Rosenfeld B. A., Zamahovsky M. P. Geometry of groups of Lie. Symmetric, parabolic and periodic spaces. Moscow, MCCME, 2003. (In Russian)

[16] Young J. W., Projective Geometry. The Open Court Publishing Company, Chicago, Illinois, 1930.

\section{Affiliations}

LYUDMILA N. ROMAKINA

ADDRESS: Saratov State University, Dept. of Geometry, Russia.

E-MAIL: romakinaln@mail.ru 